\title{
ACCELEROMYOGRAPHY- A TWITCH IN TIME SAVES LIVES
}

\author{
Davis Cherian'1, Justin Parathazham James², Susan Thomas³, Raj Sahajanandan ${ }^{4}$
}

1Postgraduate Registrar, Department of Anaesthesiology, Christian Medical College, Vellore. Tamilnadu, India.

${ }^{2}$ Assistant Professor, Department of Anaesthesiology, Christian Medical College, Vellore. Tamilnadu, India.

${ }_{3}^{3}$ Assistant Professor, Department of Anaesthesiology, Christian Medical College, Vellore. Tamilnadu, India.

4Professor, Department of Anaesthesiology, Christian Medical College, Vellore. Tamilnadu, India.

\begin{tabular}{l}
\hline ABSTRACT \\
BACKGROUND \\
Residual neuromuscular blockade (RNMB) after the use of neuromuscular blocking agents (NMBAs) increases postoperative \\
morbidity and mortality. The incidence of RNMB associated with the unmonitored use of NMBAs in our population has not been \\
ascertained. We undertook this study to determine the occurrence of RNMB, defined as train of four (TOF) ratio less than one and \\
to identify the risk factors associated with it.
\end{tabular}

\section{METHODS}

This was a prospective observational study consisting of patients who received NMBAs and underwent elective surgeries under general anaesthesia. They were monitored with TOF-Watch® SX for RNMB in the post anaesthesia care unit (PACU). RNMB was recorded and confirmed. Demographic and clinical data related to anaesthesia care, surgical procedure and post anaesthesia adverse effects were analysed.

\section{RESULTS}

A total of 195 patients who underwent elective surgeries under general anaesthesia were included in the study. The incidence of residual neuromuscular blockade was $31 \%$ with a TOF ratio of $<0.9$ and $51 \%$ with a TOF ratio of $<1$. RNMB occurred more frequently among the female gender, body mass index (BMI) $>25$ and hypothermic patients. Residual neuromuscular blockade was shown to be associated with increased use of airway adjuncts in the PACU and commonly used clinical tests were not sensitive to pick up RNMB.

\section{CONCLUSIONS}

The significant incidence of RNMB in the PACU emphasizes the need for anaesthesiologists to be mindful of this potentially serious event. Use of neuromuscular monitor can aid in the detection of RNMB and should be considered especially for the high-risk group. Detection will allow appropriate interventions before serious adverse events occur.

\section{KEY WORDS}

Neuromuscular Blocking Agents, Residual Neuromuscular Blockade, Train of Four Ratio

HOW TO CITE THIS ARTICLE: Cherian D, James JP, Thomas S, et al. Acceleromyography- a twitch in time saves lives. J. Evolution Med. Dent. Sci. 2019;8(25):2023-2027, DOI: 10.14260/jemds/2019/445

\section{BACKGROUND}

Neuromuscular blocking agents (NMBAs) are widely used in anaesthesia and though the newer NMBAs provide rapid onset and offset of effects and predictable recovery, RNMB is a reality and major contributor of the anaesthesia related morbidity and mortality.(1) Most clinicians evaluate neuromuscular function before tracheal extubation by using clinical tests of muscle strength.(2) However, these clinical tests need patient cooperation and can be performed in the presence of significant degrees of residual neuromuscular blockade.(3) The visual or tactile assessment of response to nerve stimulation done in clinical practice is subjective and does not exclude residual curarization. $(4,5)$

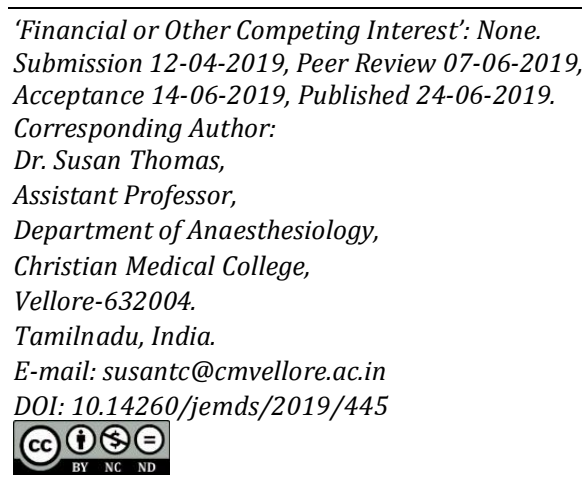

Objective monitoring of neuromuscular function may be done using electromyography (EMG), mechanomyography (MMG) or the less cumbersome acceleromyography. Acceleromyography can be performed by stimulation of the ulnar nerve and monitoring the acceleration response of the adductor pollicis. Conventionally, a Train of Four (TOF) ratio of $<0.7$, quantitatively measured using either EMG or MMG, has been considered to represent inadequate neuromuscular recovery. This value was derived from several studies published in the 1970s. ${ }^{(6-8)}$ More recent data suggested TOF ratios must recover to values $>1$, to ensure adequate recovery, which is currently taken as the gold standard. $(9,10)$ An unpublished data from Vellore, India in 2000, showed an incidence of RNMB of $28 \%$ with a TOF ratio of 0.7 . The choice of anaesthesia drugs and ventilation modes have changed since then. Given the lack of adequate data in our population and the concerns about patient safety, we conducted this study to evaluate the incidence of residual neuromuscular blockade and the risk factors associated with its use.

\section{METHODS \\ General}

Prospective evaluation of RNMB and associated risk factors was done in the PACU for patients undergoing surgery under general anaesthesia, of a tertiary referral hospital in South 
India. Institutional Review Board (IRB Min No 8940[Observe]) and Ethics committee approval was given, and the study was internally funded by the Fluid Research Grant. This manuscript adheres to the Strengthening the Reporting of Observational Studies in Epidemiology (STROBE) guidelines.

Consenting subjects of more than 18 years of age, belonging to ASA class 1 to 3, who received NMBAs for elective surgical procedures under general anaesthesia were included. Exclusion criteria were patient refusal or inability to give consent, ages $<18$ years and $>80$ years, patients with pre-existing neuromuscular disease, patients who received long acting muscle relaxants and those planned for postoperative ventilation. The patients received standard care during the intraoperative period. The conduct of anaesthesia including the choice of muscle relaxant, reversal and criteria for extubation, was at the discretion of the attending anaesthesiologist. Demographic and other clinical data such as surgery performed, type of anaesthetic, duration of surgery was recorded for each patient.

All patients on arrival into the PACU, along with routine monitoring of vital signs also had oxygen saturation measured by standard plethysmography and temperature by a digital thermometer in the axilla. As was the standard institutional practice, all patients after general anaesthesia, received supplemental oxygen by face mask at six liters per minute. The patient was then evaluated for ability to sustain head lift and hand grip for five seconds. The presence of airway adjunct on arrival to the PACU was noted. Any noisy breathing or signs of upper airway obstruction like tracheal tug or snoring were observed and corrected with airway manoeuvres or airway adjuncts.

\section{Detection of RNMB}

Detection of residual curarization was performed using a TOF-Watch ${ }^{\circledR}$ SX monitor, (Organon, Ireland Ltd., Drynam Road, Swords, Co. Dublin. Ireland), that works by means of acceleromyography. The patients' arm was placed extended on a cushioned arm board and fastened with 2-inch Velcro straps around the forearm and four fingers and free mobility of the thumb was ensured. Two surface electrodes were applied, after cleaning the volar aspect of the forearm. The distal electrode was placed proximal to the flexion crease of the wrist, as it crosses the radial side of the tendon to the flexor carpi ulnaris muscle. The second electrode was placed 2 to $3 \mathrm{~cm}$ proximal to the distal electrode. The TOF-Watch $\AA$ SX was calibrated at $30 \mathrm{~mA}$ stimulus intensity in the calibration mode 1 of the watch and measurements were taken 15 seconds apart until 2 consecutive values were within $10 \%$ of each other. The average of the two values was taken as the TOF ratio and rounded off to the lower whole number. If TOF ratio was less than 1 , readings were taken 10 minutes apart till it became greater than 1 . The incidence of TOF ratio $<1$ was calculated. Demographic variables and secondary outcomes were compared in the two groups. Intraoperative details were noted from the anaesthesia record and time to discharge was noted from the patient record register. The duration of surgery, choice of relaxant, total dose of relaxant, time from last dose to reversal of paralysis, time from reversal to extubation, dose of reversal was compared. Duration of PACU stay was the time interval between the patient coming into recovery room to the time at which the patient left, as recorded by their nurses who transferred them.

If RNMB was suspected or if any signs of upper airway obstruction were present, the concerned anaesthetist was notified, and the event was noted in the anaesthetic record. Adverse respiratory event was defined as oxygen saturation < $90 \%$ while on supplemental oxygen by face mask at $6 \mathrm{l} / \mathrm{min}$, upper airway obstruction, noisy breathing requiring chin lift, jaw thrust, use of airway adjuncts and re-intubation. Interventions were performed at the discretion of the attending anaesthesiologist and additional 0.02 microgram per $\mathrm{kg}$ of neostigmine was given up to a maximum of 0.07 microgram per $\mathrm{kg}$.

\section{Statistical Analysis}

The Statistical analyses were performed by using Statistical Package for the Social Sciences version 18, (SPSS Inc., Chicago, Illinois, USA). Patient demographics and quantitative variables were expressed in terms of mean \pm standard deviation. All categorical variables were reported using frequencies and percentages. If the distributional assumption satisfied normality, then the measurements were expressed as mean and standard deviations. If there any doubt of normality, Shapiro Wilk's test was performed to check for normality assumption and then median with inter quartile range was reported. If the assumption of normality held good, the comparison of the two groups was done using t-test. For skewed data, Mann Whitney U test was used to compare between the groups. ANOVA was used to compare more than two groups. Chi-square test was done to find the association between outcome and their risk factors. Multivariate analysis was done using multiple logistic regressions. A $p$ value $<0.05$ was considered statistically significant.

\section{RESULTS}

Between February 2015 and August 2015,195 consenting patients scheduled for elective surgical procedures under general anaesthesia and received NMBAs, were recruited for the study and monitored in the PACU for RNMB using TOF Watch ${ }^{\circledR}$ SX. Of these, 8 (4.1\%) patients were excluded from the final analysis as the TOF ratio could not be measured due to shivering or lack of patient cooperation. The demographics of the remaining 187 patients are shown in Table 1 and the incidence of residual neuromuscular block as defined by various TOF ratios are in Table 2. For further analysis the study population was divided based on TOF ratio, by the presence of RNMB (TOF <1) and absence of RNMB (TOF $>/=$ 1). By this criteria, 96 subjects had RNMB identified, making the overall incidence proportion $51 \%$.

Age was also not identified as a risk factor, since the incidence of RNMB in the $<60$ years and $>60$ years was $52.9 \%$ vs $51.2 \%, \mathrm{P}=0.89$. There was an increased incidence of RNMB in females as compared to males $(63.9 \% \mathrm{~V} / \mathrm{s} 45.2 \%)$ and the difference was statistically significant $(\mathrm{P}=0.016)$. The weight of two patients was not recorded as they were unable to stand, the relationship of $\mathrm{BMI}$, of the remaining 185 participants, with the TOF ratio was documented. The incidence of RNMB among the obese and overweight group was $12(65.1 \%)$ and $28(66.7 \%)$ was significantly higher than the normal BMI group 56 (45.2\%), p value 0.033 .

The incidence of RNMB was 73(50\%), 21(55.3\%) and 2(66.7\%) among ASA I, II and III respectively, $\mathrm{P}=0.79$. 
Patient Demographics

\begin{tabular}{|c|c|c|c|}
\hline Characteristic & $\begin{array}{c}\text { Patients with } \\
\text { RNMB (TOF<1) }\end{array}$ & $\begin{array}{c}\text { Patients with No } \\
\text { RNMB }\end{array}$ & p Value \\
\hline $\begin{array}{c}\text { Age (y) } \\
<60\end{array}$ & $9(52.94 \%)$ & $8(47.06 \%)$ & 0.89 \\
$>=60$ & $87(51.18 \%)$ & $83(48.82 \%)$ & \\
\hline Gender & $57(45.2 \%)$ & $69(54.8 \%)$ & 0.016 \\
Male & $39(63.9 \%)$ & $22(36.1 \%)$ & \\
Female & $73(50 \%)$ & $73(50 \%)$ & \\
\hline ASA & $21(55.3 \%)$ & $17(44.7 \%)$ & 0.79 \\
I & $2(66.7 \%)$ & $1(33.3 \%)$ & \\
II & & & \\
III & $56(45.2 \%)$ & $68(54.8 \%)$ & \multirow{2}{*}{0.033} \\
BMI & $28(65.1 \%)$ & $15(34.9 \%)$ & \\
Normal & $12(66.7 \%)$ & $6(33.3 \%)$ & \\
Overweight & & & \\
Obese & & & \\
\hline & & & \\
\hline
\end{tabular}

Table 1.

Abbreviations: Y- Years, ASA- American Society of Anesthesiologists, BMI- Body Mass Index

\begin{tabular}{|c|c|c|}
\hline TOF Ratio Less Than & Number of Participants N= 187 & $\%$ \\
\hline 0.7 & 4 & 2.13 \\
0.9 & 59 & 31.55 \\
1 & 96 & 51.33 \\
\hline \multicolumn{2}{|c|}{ Table 2. Incidence of RNMB } \\
\hline
\end{tabular}

\begin{tabular}{|c|c|c|c|}
\hline Risk Variable & Odds Ratio & 95\% CI & p-Value \\
\hline $\begin{array}{c}\text { Gender } \\
\text { Male }\end{array}$ & 1.00 & & \\
Female & 2.17 & $1.06-4.45$ & 0.03 \\
\hline $\begin{array}{c}\text { BMI } \\
\text { Normal }\end{array}$ & 1.00 & & \\
Overweight/Obese & 2.13 & $1.07-4.25$ & 0.03 \\
\hline Relaxant & 1.00 & & \\
Atracurium & 1.97 & $0.67-5.79$ & 0.22 \\
Vecuronium & 1.65 & $0.11-25.28$ & 0.72 \\
Rocuronium & 1.01 & $0.98-1.04$ & 0.35 \\
\hline Total Dose & & \\
Time interval between & 0.998 & $0.99-1.004$ & 0.45 \\
Last dose to reversal & 0.997 & $0.97-1.02$ & 0.725 \\
Reversal to measurement & 1.001 & $0.99-1.005$ & 0.827 \\
Total procedure time & 8.88 & $1.63-48.32$ & 0.01 \\
\hline $\begin{array}{c}\text { Temperature } \\
<35\end{array}$ & 1.00 & & \\
$\geq 35$ & Regression & \\
\hline Table 3. Multivariable Analysis of Risk Factors by Logistic \\
$\quad$
\end{tabular}

Of the intraoperative variables' isoflurane was the most commonly used agent for maintenance and there was no significant difference in the incidence of RNMB between agents, isoflurane (50.8\%), sevoflurane (71.4\%) and desflurane (33.3\%) ( $\mathrm{p}=0.46)$.

Use of succinylcholine prior to the use of nondepolarizing muscle relaxants showed a higher frequency of residual neuromuscular block (65.7\%) compared to when it was not used (48\%) but the difference was not significant $(\mathrm{P}=0.056)$.

Vecuronium was relaxant used intra operatively in 108 participants (57.8\%), Atracurium in 75 (40.1\%) and Rocuronium in $4(2.1 \%)$. The incidence of RNMB was not significantly different in atracurium and vecuronium groups. The total dose of the relaxant given and the total dose per kilogram body weight was also compared with the presence or absence of residual block and there was no statistical significance. There was no significant correlation between the total dose of Neostigmine given or Neostigmine dose calculated to per kg body weight.

The various time intervals such as procedure time, time of last RNMB to reversal, time of reversal to measurement of the
TOF ratio, were comparable between the two groups. The mean time interval from reversal to extubation was $12.7 \pm 6.6$ minutes in all patients and the mean time from extubation to measurement of TOF ratio was $24.8 \pm 10.2$ minutes which was comparable in those with and without RNMB $(23.9 \pm 8.50$ vs. $25.7 \pm 11.7$ ).

The patients with TOF $<1$ required the use of airway adjuncts more than the patients with TOF $\geq 1$ and this was statistically significant $(65.4 \%$ Vs $34.6 \%, P=0.017)$ and they were more likely to be shifted to the PACU with the airway adjunct. The incidence of hypothermia in our PACU was $6.9 \%$. The hypothermic patients had a significantly higher chance of having residual neuromuscular blockade $(84.6 \%)$ as compared to non-hypothermic patients (48.9\%) ( $\mathrm{P}=0.013)$.

The duration of time spent in the post anaesthesia care unit (PACU), by the TOF $<1$ group was $109.79 \pm 47.98$ minutes as compared to $96.88 \pm 48.58$ minutes by the TOF $\geq 1$ group and this was difference was not statistically significant.

Clinical tests such as sustained head lift for $>5$ seconds, sustained hand grip $>5$ seconds were compared with the acceleromyographic TOF ratio in their sensitivity, specificity, positive and negative predictive value to detect residual neuromuscular block. The inability to sustain hand grip for $>$ 5 seconds was more specific than sustained head lift $>5$ seconds for predicting absence of RNMB.

\section{DISCUSSION}

The present study was performed to assess the incidence of RNMB in the PACU in subjects who received NMBAs for facilitation of endotracheal intubation and surgical access. Despite better understanding of the neuromuscular physiology and pharmacology, it is evident from our results that the incidence of RNMB is a frequent problem in the recovery room and is associated with significant morbidity. For our analysis, we took TOF ratio of less than 1 as indicative of residual neuromuscular block. Since acceleromyography (AMG) frequently overestimated TOF, to reliably exclude a residual neuromuscular block, a TOF ratio of greater than 1 has to be present. $(3,11-13)$

The incidence of residual curarization (TOF ratio $<1$ ) in our recovery, was found to be $51.3 \%$, in 187 patients comparable to studies done among other study populations, that reported incidence of $31 \%$ to $50 \% \cdot(4,5,13)$ In the metanalysis done by Naguib et al, the incidence of residual neuromuscular blockade varied from 5 to $93 \%$, and the pooled incidence of residual neuromuscular blockade with a TOF ratio less than 0.9 , in intermediate acting neuromuscular drugs, was $41 \%$.(14) Our results also matched the study done by Kumar et al whose incidence was $57 \%$ with TOF ratio of 0.9.(15)

Analysis of demographic data showed a statistically significant increase in the incidence of RNMB among females, this contrasted with a study done in Australia residual curarization were more among the males.(16) BMI greater than 25 was shown to be a risk factor as evidenced by the increased incidence of RNMB due to overdosing and delayed clearance.(17),(18) The cause of this finding they attributed to dosing of the NMBD based on real body weight and not ideal body weight leading to more redistribution.

The frequency of RNMB appeared to be more in the Sevoflurane group since inhalational anaesthetics potentiate the action of neuromuscular blocking drugs, recovery was 
slower in inhalational agents as compared to Propofol, and Sevoflurane slowed it more than Isoflurane. $(19,20)$ There were no patients who received TIVA in our study population, so its effect on reducing the incidence of RNMB could not be studied.

The use of succinylcholine before NDMR had no significant effect on the incidence of RNMB. The incidence was highest in the were rocuronium group followed by vecuronium and then the atracurium group, but our numbers too small to derive any significant conclusion. When relaxant subgroups were analysed based on the total cumulative dose and the total dose per $\mathrm{kg}$ body weight, no statistically significant differences were seen. Residual neuromuscular block was more with rocuronium as compared to vecuronium as compared to cisatracurium.(21),(22) Definitive evidence implicating one intermediate acting relaxant over other has not emerged, but residual block is more often in long acting relaxants as compared to intermediate acting relaxants, which has led to their reduced usage in clinical practice.(14)

All our patients usually received $2.5 \mathrm{mg}$ of neostigmine irrespective of last dose, also no differences were observed in the mean dose of reversal and mean dose per kilogram in patients with and without neuromuscular blockade. We did not have any data on timing of reversal based on qualitative or quantitative monitoring as it was not a routine practice in our setup. The timing of reversal is essential because the excess neostigmine itself can lead to neuromuscular weakness, manifesting as fade in TOF ratio, hence the dose given must be modified according to objective neuromuscular monitoring.(23)

The duration of procedure, time from last relaxant to measurement, time of reversal to measurement had no statistical significance in the incidence of residual neuromuscular block. This contrasts with Yip et al, who found that the incidence of RNMB was more in shorter procedures.(16) The time from last dose of relaxant to reversal was longer and the average time between reversal to extubation was less than the recommended time of 15 to 30 minutes before the planned extubation as compared to other studies. $(20,24)$ This may be due to the practice of using PSV Pro mode of ventilation in the GE anaesthesia machines towards the end of the procedure. The depth of neuromuscular blockade at which they were reversed is unknown as the TOF count or ratio at reversal was not monitored.

In our study, the incidence of airway related events and the use of airway adjuncts was significantly higher among the participants with a TOF less than 1 , which was similar to other studies.(25) Though hypoxia has been reported in the PACU, in patients with RNMB, they had measured oxygen saturation in the recovery and instituted oxygen therapy if there was desaturation.(26) Our practice was to start all patients on oxygen and discontinue it, if they were able to maintain desirable oxygenation after immediate recovery. We did not change this practice as ours was an observational study. This might have masked the true incidence of hypoxia in our study population.

The mean time spent by patients with RNMB was not significantly more than the patients without RNMB. A similar study done by Butterly et al found a statistical difference among time to discharge from recovery room whereas Yip et al did not.(16,21) Many factors affected the total time spent by our patient in the recovery, recovery room nurses call the respective ward once the patient meets the criteria for discharge according to Aldrete's score. The availability of the ward attendant, ward nursing staff and distance of the ward from the recovery room were contributing factors for the delay in actual discharge of the patient. The time to eligibility of discharge could have been better correlated with the incidence of residual neuromuscular blockade.

Post-operative hypothermia, with an axillary temperature less than 35 degree Celsius, was found to be a significant factor affecting the incidence of residual neuromuscular blockade. Hypothermia potentiates the action of neuromuscular blockers and causes delayed recovery. $(27,28)$ Temperature was not monitored intra operatively in $30 \%$ of our subjects.

Our study aimed at finding the possible risk factors for RNMB and univariate analysis showed female sex, high BMI and hypothermia as risk factors for RNMB and multivariate analysis using logistic regression showed that these factors were significant predictors as well. The chances of having RNMB were doubled in females compared to males (Odds Ratio 2.17, p value 0.03 ) and overweight/obese individuals as compared to their normal counterparts. (Odds Ratio 2.13, p value 0.03$)$. Temperature less than 35 increased the chance of RNMB by eight times. (OR 8.8, p value 0.01 ).

Clinical tests of muscle strength are not very reliable in detecting residual neuromuscular block. Our findings also suggest that the ability to sustained head lift and hand grip for 5 seconds are not enough to exclude residual neuromuscular block. Sustained hand grip appeared to more specific than head lift in predicting absence of neuromuscular block which are like results obtained by Cammu et al.(4)

\section{Limitations}

Ours was a prospective observational study. The study was not designed to identify the individual risk factors. Time to adequate criteria for discharge may have been better parameter than total time spent in recovery to correlate the impact of RNMB on patients in the recovery. Intra operative variables were documented from the anaesthetic record of the patient, so the reliability of the data also depended on the accuracy of monitoring. Ideally, a baseline TOF ratio should be available, prior to administration of muscle relaxants, to normalize the TOF ratio before extubation. This would have created a bias in our study by alerting the anaesthetist and hence was not done. All observations were made by one primary investigator, so bias may be present. The clinical tests were done prior to observing the TOF ratio to minimize the bias. The TOF watch SX is highly sensitive to movement artefacts causing wide variations in successive TOF ratios observed in the same patient. Calibrating the TOF watch improved the precision, but sometimes multiple attempts of calibration had to be made on the same patient. This process becomes more difficult in the immediate post-operative period when patients are drowsy, disoriented, shivering and sometimes uncooperative.

\section{CONCLUSIONS}

The incidence of RNMB in the PACU, using the current recommendation of TOF ratio $\geq 1$ using acceleromyography, is a significant $51 \%$ and anaesthesia professionals should have a high index of suspicion for the same. Routine use of monitoring devices like the TOF Watch SX will be helpful in 
detecting RNMB, especially in the high-risk population. Female sex and patients with BMI > 25 and hypothermia were identified as risk factors for developing residual neuromuscular block. The use of devices to detect RNMB will allow prompt pharmacological treatment, optimal use of airway adjuncts, prevention of airway compromise and improved patient outcomes.

\section{REFERENCES}

[1] Viby-Mogensen J, Jørgensen BC, Ording H. Residual curarization in the recovery room. Anesthesiology 1979;50(6):539-41.

[2] Grayling M, Sweeney BP. Recovery from neuromuscular blockade: a survey of practice. Anaesthesia 2007;62(8):806-9.

[3] Eikermann $M$, Groeben $H$, Hüsing $J$, et al. Accelerometry of adductor pollicis muscle predicts recovery of respiratory function from neuromuscular blockade. Anesthesiology 2003;98(6):1333-7.

[4] Cammu G, De Witte J, De Veylder J, et al. Postoperative residual paralysis in outpatients versus inpatients. Anesth Analg 2006;102(2):426-9.

[5] Hayes AH, Mirakhur RK, Breslin DS, et al. Postoperative residual block after intermediate-acting neuromuscular blocking drugs. Anaesthesia 2001;56(4):312-8.

[6] Ali HH, Kitz RJ. Evaluation of recovery from nondepolarizing neuromuscular block, using a digital neuromuscular transmission analyzer: preliminary report. Anesth Analg 1973;52(5):740-5.

[7] Ali HH, Wilson RS, Savarese JJ, Kitz RJ. The effect of tubocurarine on indirectly elicited train-of-four muscle response and respiratory measurements in humans. Br J Anaesth 1975;47(5):570-4.

[8] Brand JB, Cullen DJ, Wilson NE, et al. Spontaneous recovery from non-depolarizing neuromuscular blockade: Correlation between clinical and evoked responses. Anesth Analg 1977;56(1):55-8.

[9] Sundman E, Witt H, Olsson R, et al. The incidence and mechanisms of pharyngeal and upper esophageal dysfunction in partially paralyzed humans: pharyngeal videoradiography and simultaneous manometry after atracurium. Anesthesiology 2000;92(4):977-84.

[10] Eriksson LI, Sundman E, Olsson R, et al. Functional assessment of the pharynx at rest and during swallowing in partially paralyzed humans: simultaneous videomanometry and mechanomyography of awake human volunteers. Anesthesiology 1997;87(5):1035-43.

[11] Capron F, Fortier LP, Racine S, et al. Tactile fade detection with hand or wrist stimulation using trainof-four, double-burst stimulation, 50-hertz tetanus, 100-hertz tetanus and acceleromyography. Anesth Analg 2006;102(5):1578-84.

[12] Capron F, Alla F, Hottier C, et al. Can acceleromyography detect low levels of residual paralysis? A probability approach to detect a mechanomyographic train-of-four ratio of 0.9 . Anesthesiology 2004;100(5):1119-24.

[13] Debaene B, Plaud B, Dilly MP, et al. Residual paralysis in the PACU after a single intubating dose of non- depolarizing muscle relaxant with an intermediate duration of action. Anesthesiology 2003;98(5):1042-8.

[14] Naguib M, Kopman AF, Ensor JE. Neuromuscular monitoring and postoperative residual curarisation: a meta-analysis. Br J Anaesth 2007;98(3):302-16.

[15] Kumar GV, Nair AP, Murthy HS, et al. Residual neuromuscular blockade affects postoperative pulmonary function. Anesthesiology 2012;117(6):1234-44.

[16] Yip PC, Hannam JA, Cameron AJ, et al. Incidence of residual neuromuscular blockade in a post-anaesthetic care unit. Anaesth Intensive Care 2010;38(1):91-5.

[17] Weinstein JA, Matteo RS, Ornstein E, et al. Pharmacodynamics of vecuronium and atracurium in the obese surgical patient. Anesth Analg 1988;67(12):1149-53.

[18] Joshi SB, Upadhyaya KV, Manjuladevi M. Comparison of neostigmine induced reversal of vecuronium in normal weight, overweight and obese female patients. Indian J Anaesth 2015;59(3):165-70. http://www.ijaweb.org/article.asp?issn=0019-

[19] Reid JE, Breslin DS, Mirakhur RK, et al. Neostigmine antagonism of rocuronium block during anaesthesia with sevoflurane, isoflurane or propofol. Can J Anaesth 2001;48(4):351-5.

[20] Kim KS, Cheong MA, Lee HJ, et al. Tactile assessment for the reversibility of rocuronium-induced neuromuscular blockade during propofol or sevoflurane anaesthesia. Anesth Analg 2004;99(4):1080-5.

[21] Butterly A, Bittner EA, George E, et al. Postoperative residual curarization from intermediate-acting neuromuscular blocking agents delays recovery room discharge. Br J Anaesth 2010;105(3):304-9.

[22] Khan S, Divatia JV, Sareen R. Comparison of residual neuromuscular blockade between two intermediate acting nondepolarizing neuromuscular blocking agents - rocuronium and vecuronium. Indian J Anaesth 2006;50(2):115-7.

[23] Kopman AF, Eikermann M. Antagonism of nondepolarising neuromuscular block: current practice. Anaesthesia 2009;64 Suppl 1:22-30.

[24] Kirkegaard H, Heier T, Caldwell JE. Efficacy of tactileguided reversal from cisatracurium-induced neuromuscular block. Anesthesiology 2002;96(1):4550 .

[25] Murphy GS, Szokol JW, Marymont JH, et al. Intraoperative acceleromyographic monitoring reduces the risk of residual neuromuscular blockade and adverse respiratory events in the post-anaesthesia care unit. Anesthesiology 2008;109(3):389-98.

[26] Murphy GS, Szokol JW, Marymont JH, et al. Residual neuromuscular blockade and critical respiratory events in the postanaesthesia care unit. Anesth Analg 2008;107(1):130-7.

[27] Caldwell JE, Heier T, Wright PM, et al. Temperaturedependent pharmacokinetics and pharmacodynamics of vecuronium. Anesthesiology 2000;92(1):84-93.

[28] Leslie K, Sessler DI, Bjorksten AR, et al. Mild hypothermia alters propofol pharmacokinetics and increases the duration of action of atracurium. Anesth Analg 1995;80(5):1007-14. 